\title{
Erratum to: CME instructions: Cardiac imaging for the assessment of patients being evaluated for kidney or liver transplantation
}

\section{ERRATUM TO: J NUCL CARDIOL 2015; 22:279-81 DOI 10.1007/S12350-015-0093-8}

In the CME instructions article referenced above, the primary author was listed incorrectly under the disclosure section. The corrected CME instructions are below. We apologize for this oversight.

\section{STATEMENT OF NEED}

The following gaps in knowledge have been identified in clinical practice demonstrating the learner's need to acquire the skills and strategies presented in this CME activity.

1. Due to the scarcity of available organs, the selection of appropriate transplant recipients with an acceptable cardiovascular risk profile is critical.

2. A recent AHA/ACC consensus statement on the management of these patients has been proposed.

3. There is a high burden of cardiovascular disease in patients with end-stage renal disease and end-stage liver disease.

4. There is current controversy regarding the use of non-invasive cardiac imaging tools to guide cardiovascular risk assessment and management of kidney and liver transplant candidates.

\section{TARGET AUDIENCE}

This activity is targeted at imaging professionals and is intended to provide the latest information on

The online version of the original article can be found under doi: 10.1007/s12350-015-0093-8.

J Nucl Cardiol 2015;22:782-4.

$1071-3581 / \$ 34.00$

Copyright (C) 2015 American Society of Nuclear Cardiology.

doi:10.1007/s12350-015-0207-3 clinical practice and cutting-edge scientific advances in nuclear cardiology and cardiac imaging.

\section{OVERALL PURPOSE}

The purpose of this CME activity in the Journal of Nuclear Cardiology is to increase the learners' competence in the application of nuclear cardiology strategies in clinical practice.

\section{OBJECTIVES}

After reading and reflecting upon this article in the Journal Nuclear Cardiology, the learner should be able to:

1. Understand the importance of coronary disease surveillance in patients being evaluated for kidney or liver transplantation.

2. Know the advantages and limitations of different non-invasive screening modalities for cardiovascular disease and effectively select the appropriate tool based on patient characteristics.

3. Describe the AHA/ACC consensus statement recommendations related to the use of non-invasive cardiac imaging of patients being evaluated for kidney or liver transplantation.

\section{ACCREDITATION AND CONTINUING EDUCATION CREDIT}

\section{Physicians}

The American Society of Nuclear Cardiology is accredited by the Accreditation Council for Continuing Medical Education to provide continuing medical education for physicians.

The American Society of Nuclear Cardiology designates this Journal-based CME activity for a maximum of 1 AMA PRA Category 1 Credits $^{\mathrm{TM}}$. Physicians should only claim credit commensurate with the extent of their participation in the activity. 


\section{Technologists}

The American Society of Nuclear Cardiology is a recognized provider of continuing education credit for technologists. ASNC's Continuing Education (ACE) credit is accepted by both NMTCB and ARRT. This Journal-based activity has been approved for a maximum of 1 ARRT Category A credits for Technologists.

\section{PRINCIPAL FACULTY AND THEIR CREDENTIALS}

CME Editor: William A. Van Decker, MD, FASNC, Temple University Hospital

Primary Author: Rami Doukky, MD, John H. Stroger, Jr. Hospital of Cook County

\section{DISCLOSURE}

As an accredited provider of the Accreditation Council for Continuing Medical Education (ACCME), the American Society of Nuclear Cardiology (ASNC) adheres to the ACCME's 2005 Standards for Commercial Support. In compliance with these standards, it is ASNC's policy to ensure balance, independence, objectivity, and scientific merit in all of its educational activities through the disclosure of relevant financial relationships with commercial companies and resolution of conflicts of interest. The financial interest or relationships requiring disclosure are outlined in ASNC's CME Conflict of Interest Policy. All persons who influenced the content of this activity are required to disclose any relevant financial relationships. The American Society of Nuclear Cardiology has reviewed these disclosures and resolved or managed all identified conflicts of interest through a peer review process.

The following author who was involved in the development of this activity reported the following financial relationships: Rami Doukky, MD, Grant Support-Astellas Pharma; Advisory Board-Astellas Pharma.

The following members of the JNC Editorial Staff and ASNC staff who were involved in the planning and development of this activity reported no financial relationships: William Van Decker, MD, FASNC and Wendy Passerell.

The following question/objective reviewer(s) who was involved in the development of this activity reported the following financial relationships: Karthik Ananthasubramaniam, MD, Grant Support-Astellas Pharma US, GE Healthcare, Alnylam Pharmaceuticals.

The following question/objective reviewer(s) who was involved in the development of this activity reported no financial relationships: Maria Sciammarella, MD.

\section{OFF-LABEL USE}

Articles may include discussion of drugs or devices, or uses of drugs or devices, that have not been approved by the Food and Drug Administration (FDA) or have been approved by the FDA for specific uses only. The FDA has stated that it is the responsibility of the physician to determine the FDA clearance status of each drug or device he or she wishes to use in clinical practice. ASNC is committed to the free exchange of medical education. Inclusion of any discussion in this program, including discussion on off-label uses, does not imply an endorsement by ASNC of the uses, products, or techniques presented.

\section{CONTINUING EDUCATION TERM OF APPROVAL}

Release Date: March 8, 2015

Expiration Date: March 7, 2016

\section{METHOD OF PARTICIPATION}

To receive a statement of credit, participants must successfully complete the post-test quiz and evaluation questions after reading and reflecting on the article. The participant selects the single most appropriate answer for each post-test question. A score of $75 \%$ or higher is needed to pass the post-test quiz. If less than $75 \%$ of the questions were correct, the participant will be notified and may resubmit the quiz with modified answers up to three times.

Estimated time of completion is 1 hour.

To complete the post-test and evaluation, please go to http://www.asnc.org/content_11624.cfm.

\section{BIBLIOGRAPHY}

Bibliographic sources are cited throughout the article and a full bibliography is provided at the end of the article to provide you with further study resources on this topic.

\section{MEDIUM OR COMBINATION OF MEDIUM USED}

\author{
Internet Explorer (Latest Version) \\ Firefox (Latest Version) \\ Google Chrome \\ Safari (Latest Version) \\ Adobe Acrobat Reader \\ Internet Explorer is not supported on the Macintosh \\ Mac OS 10.4 \\ Windows 7 or above
}




\section{PROCESSING FEES}

ASNC members may claim continuing education credits at no charge. Non-members will be charged $\$ 50$ per activity.

\section{ACKNOWLEDGEMENT OF COMMERCIAL SUPPORT}

This activity is not supported by commercial support.

\section{PRIVACY \& CONFIDENTIALITY STATEMENT}

ASNC will record learner's personal information as provided on CME evaluations to allow for issuance and tracking of CME certificates. ASNC may also track aggregate responses to questions in activities and evaluations and use these data to inform the ongoing evaluation and improvement of its CME program. No individual performance data or any other personal information collected from evaluations will be shared with third parties.

\section{ASNC COPYRIGHT INFORMATION}

Please see the "Terms of Use" at the bottom of any asnc.org web page.

\section{AMERICANS WITH DISABILITIES ACT}

The American Society of Nuclear Cardiology supports the Americans with Disabilities Act. Please submit a written request at least two weeks before the program date, and ASNC will be glad to assist you with any special needs to make this program accessible to you. Please fax your request to the Education Department at (301) 215-7113.

\section{ACKNOWLEDGEMENT OF COMMERCIAL SUPPORT}

This activity does not have commercial support.

\section{LEARNERS' BILL OF RIGHTS}

Please click to access Learners' Bill of Rights.

\section{CONTACT INFORMATION}

For technical assistance or information, please contact CECity at lifetimesupport@ cecitysupport.com

For questions regarding CME content or obtaining CME credit, please contact the American Society of Nuclear Cardiology at info@asnc.org. 